\title{
Head and Neck Surgery During COVID-19 Pandemic: Experience from a Tertiary Care in India
}

\author{
Smriti Panda ${ }^{1} \cdot$ Saurabh Vig ${ }^{2}$. Chirom Amit Singh ${ }^{1}$ (D) Abhilash Konkimalla ${ }^{1} \cdot$ Alok Thakar $^{1} \cdot$ Pirabu Sakthivel $^{1}$. \\ Kapil Sikka ${ }^{1} \cdot$ Rajeev Kumar $^{1} \cdot$ Sushma Bhatnagar ${ }^{2} \cdot$ Anant Mohan $^{3} \cdot$ Pavan Tiwari $^{3} \cdot$ Ved Prakash Meena $^{4}$. \\ Rohit K. Garg ${ }^{4} \cdot$ Sumanth Bollu ${ }^{1} \cdot$ Kuldeep Thakur ${ }^{1}$
}

Received: 26 October 2020 / Accepted: 27 July 2021 / Published online: 12 September 2021

(C) Indian Association of Surgical Oncology 2021

\begin{abstract}
Head and neck pathologies requiring surgical intervention are considered a high-risk subsite in the context of COVID-19 pandemic by virtue of its close proximity to the mucosa of the upper aerodigestive tract. Retrospective review of all head and neck surgical procedures is undertaken during the pandemic from 23rd April 2020 to 30th September 2020. One hundred procedures were performed on 98 patients. COVID-19 status determined by SARS-Cov-2 RT-PCR at baseline was negative for 81 , positive in 8 and unknown in 11. The RT-PCR negative subgroup included 40 diagnostic procedures and 41 ablative and or reconstructive procedures for head and neck neoplasms. None of the patients or health-care workers converted to COVID-19-positive status during the duration of the hospital stay. There were no cases with 30-day mortality. Clavien-Dindo grading for postoperative complications was as follows: 1-4, 2-12, 3a-2, 3b-1. Eleven patients with unknown COVID-19 status at baseline underwent emergency tracheostomy in a COVID-19 designated operating room for upper airway obstruction secondary to head and neck cancer. Of the 8 procedures conducted on known cases of COVID-19, 6 were tracheostomies performed for COVID-19 ARDS. The rest were maxillectomy for acute invasive mucormycosis and incision and drainage for parotid abscess. A matched-pair analysis was performed with similarly staged historical cohort operated during January to December 2016 to compare peri-operative complication rates (Clavien-Dindo Score). Incidence of complication with higher Clavien Dindo Score $(>/=3 \mathrm{a})$ was found to be lower in those patients operated during the pandemic $(\mathrm{p}=0.007)$. By meticulous preoperative COVID-19 screening and isolation, head and neck surgical procedures can be continued to avoid delay in diagnosis and treatment without jeopardising the risk of transmission of COVID-19 to the patients or health-care workers.
\end{abstract}

Keywords COVID-19 $\cdot$ Head and neck cancer $\cdot$ Head and neck surgery $\cdot$ SARS-Cov-2

\section{Introduction}

Chirom Amit Singh

amitchirom@gmail.com

1 Department of Otorhinolaryngology and,Head and Neck Surgery, All India Institute of Medical Sciences, 110029 New Delhi, India

2 Department of Onco-Anesthesia, Pain and Palliative Medicine, All India Institute of Medical Sciences, New Delhi, India

3 Department of Pulmonary Medicine, All India Institute of Medical Sciences, New Delhi, India

4 Department of Medicine, All India Institute of Medical Sciences, New Delhi, India
Severe acute respiratory syndrome coronavirus 2 (SARSCoV-2) was declared as a pandemic in March 2020 by the WHO [1]. The rapid surge of COVID-19 cases resulted in the complete restructuring of the health-care system worldwide. With state-imposed lockdown, shunting of resources and manpower towards care of critically ill COVID-19 patients, there have been profound repercussions for patients suffering from cancer [2]. Multilevel challenges confronting cancer care during these times include access to health care for timely diagnosis, treatment delays with consequent disease progression, treatment disruption and hindrance towards physical follow-up and cancer surveillance [2]. This has the potential to result in the decline of survival rates with projected rates of $5-10 \%$ reduction 
predicted for high-income countries [2]. Head and neck cancer (HNC) accounts for $30-40 \%$ of all cancer cases in India [3]. The added obstacles faced by patients and health-care workers involved with HNC care arise due to the potential for the generation of aerosol during evaluation and treatment [4]. Though there was significant contention surrounding the appropriate treatment algorithm for HNC in the initial part of the pandemic, we now have numerous scientific bodies providing guidelines for the management of HNC including treatment prioritization and triage [5]. This study intends to highlight the practice of head and neck surgery being followed at our institute which is also a COVID-19 designated treatment facility with the focus on the preoperative testing strategy, steps taken to curtail COVID-19 transmission among patients and health-care workers and perioperative outcomes.

\section{Methods}

This was a retrospective study conducted on all patients who underwent elective as well as emergency head and neck surgery at the National Cancer Institute- All India Institute of Medical Sciences, Jhajjar from 23rd April 2020 to 30th September 2020. This retrospective review was exempted from Ethical Clearance by the Institutional Ethical Committee. The study was performed in accordance with the ethical standards as laid down in the 1964 Declaration of Helsinki. Informed written consent was obtained from all the participants.

\section{Preoperative Work-Up (Fig. 1a)}

All patients presenting to the out-patient department were screened by a resident doctor for symptoms consistent with COVID-19 (fever, cough, shortness of breath, upper respiratory tract infection), a recent history of travel and contact with a known case of COVID-19. In the presence of any of the above features, the consultation was deferred, and the patient was managed on the lines of a COVID-19 suspect as shown in Fig. 1a. All head and neck examinations were performed by trained Otolaryngologists with second-level personal protective equipment (PPE) ( $\mathrm{N}-95$ respirator, eye protection goggles, face shield, gown and double gloves). For the aerosol generating procedures like flexible fibre-optic endoscopy, rigid nasal endoscopy and oral cavity biopsies, SARS-Cov-2 RT-PCR was obtained $24-48 \mathrm{~h}$ before the scheduled procedure. These procedures were also conducted with second level PPE.

\section{In-Patient and Operating Room Workflow (Fig. 1b)}

Patients scheduled for elective head and neck procedures were admitted in an isolation ward after being screened for COVID-19 symptomatology. Following admission, SARSCov-2 RT-PCR was obtained. After a negative RT-PCR report, patients were shifted to the main hospital emergency ward dedicated to non-COVID-19 cancer patient care. Surgery was scheduled within $24-48$ h of a negative RT-PCR. Surgical procedures for patients detected to be positive for COVID-19 was deferred for a period of 17 days and were re-tested for SARS-Cov-2 before readmission for surgery. All head and neck surgeries were performed with second-level PPE.

Patients presenting with life-threatening head and neck emergencies, like stridor, deep neck space abscess and haemorrhage from the upper aero-digestive tract, were not deemed suitable for the above-mentioned testing strategy. Patients were instead admitted to the isolation ward for initial stabilisation. Deep nasal and oropharyngeal swabs for SARS-Cov-2 RT-PCR were collected simultaneously. Patients were operated upon on an emergent basis in a COVID-designated operating room. Health-care personnel were required to don level $3 \mathrm{PPE}$.

Emergent and semi-emergent procedures on diagnosed cases of COVID-19 were also performed in a COVIDdesignated operating room with level 3 PPE.

\section{Airway and Anaesthetic Management}

A final pre-procedure anaesthetic assessment to formulate the plan of anaesthesia and rule out any un-optimized comorbidities was done after the negative RT-PCR report for elective cases. For emergency procedures with unknown COVID-19 status, the anaesthetist used level 2 PPE for pre-procedural assessment.

Elective cases planned for definitive resections, the plan of anaesthesia was general anaesthesia with nasal intubation for the duration of the procedure and elective tracheostomy at the end of surgery. In general, video laryngoscope was used to intubate and secure the airway as per the existing guidelines for airway access in the COVID-19 pandemic.

For cases with limited or zero mouth opening, laryngeal lesions with critical airway stenosis or any other anticipated difficulties in securing the airway the choice of technique to secure the airway (awake fibreoptic nasal intubation or pre-procedural tracheostomy) were left on the discretion of individual anaesthetist in charge for the case. All elective cases at the end of surgery were reversed from neuromuscular blockade, awakened from anaesthesia 
Fig. 1 a. Workflow and screening strategy for a patient presenting to the out-patient department for head and neck surgery consultation. b Workflow and screening strategy for patients requiring elective and emergency head and neck surgery procedures a

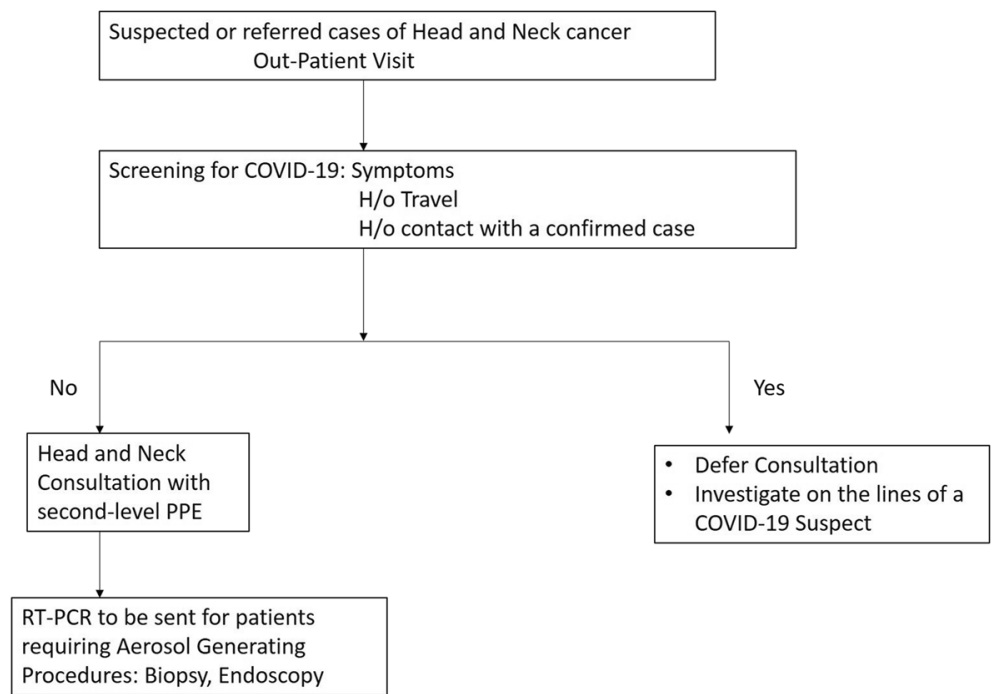

b

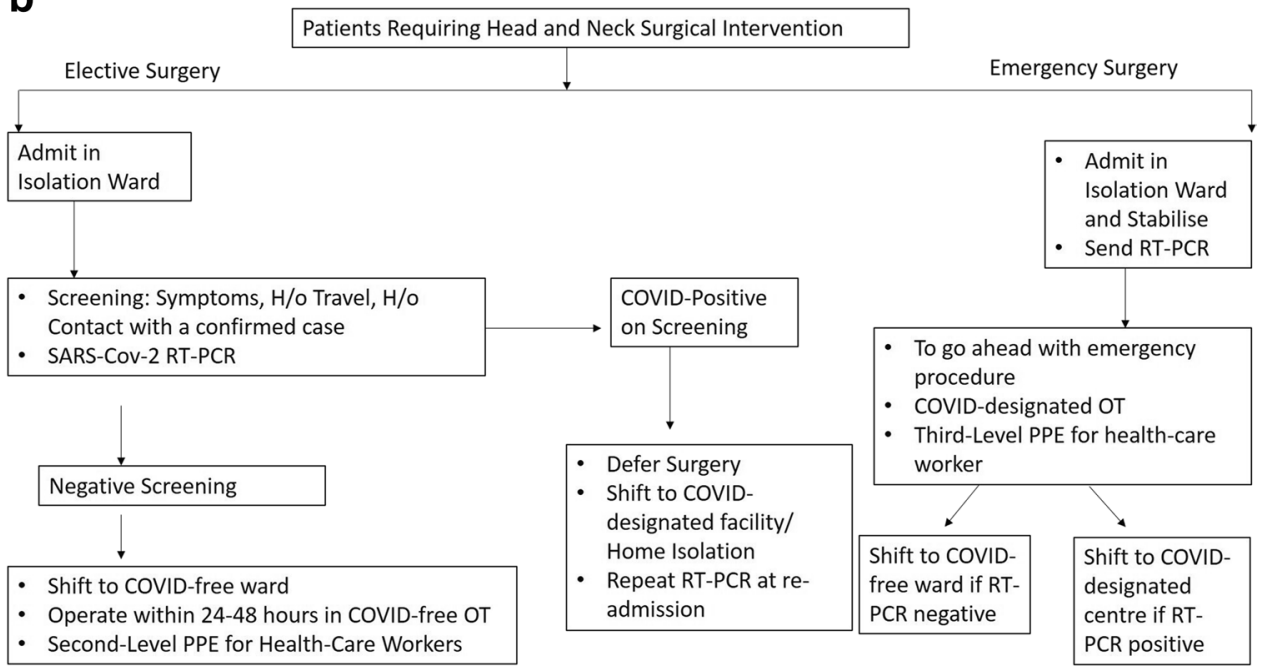

and shifted to the emergency ward on CPAP (continuous positive airway pressure) support with spontaneous breathing. Patients were shifted to T-piece on the morning of the first postoperative day.

For laryngeal biopsies or evaluation under anaesthesia, the airway was secured with a microlaryngeal surgery tube. In COVID-19-positive patients with ARDS for tracheostomy, ventilatory and sedation protocols followed in the ICU were continued for the duration of the procedure.

\section{Variables Analysed}

The COVID-19 status of all patients was categorized as positive, negative and unknown. Patients who underwent major head and neck procedures were evaluated for their performance status and comorbidities using the 11-point modified frailty index [6]. The perioperative outcomes were assessed using the Clavien-Dindo score for postoperative complications [7], 30-day hospital readmission rate, 30-day mortality, time taken for decannulation and initiation of oral feeds.

\section{Results}

\section{Preoperative Screening}

One hundred head and neck surgery procedures were performed during the 21 weeks period from 23rd April 2020, to 30th September 2020, on 98 patients (Table 1). Baseline parameters have been summarised in Table 1. Preoperative COVID-19 status at the time of surgery was positive in 8 , negative in 79 and unknown in 11 . Of the 81 patients scheduled for elective procedures, 2 were incidentally detected to be positive on preoperative SARS-Cov-2 RT-PCR 
Table 1 Baseline characteristics
Number of procedures

Age (years)

$52.04 \pm 13.6$

Sex

Male

Female

Preoperative COVID-19 status

Positive

8

Negative

79

Unknown

Anaesthesia

GA

LA

Elective procedures with baseline negative RT-PCR ( 81 procedures)

Diagnostic

Therapeutic

Subsites for ablative/reconstructive procedures $(n=39)$

Gingivobuccal complex

Lateral border tongue

Maxilla

Larynx and hypopharynx

Floor of mouth

Parotid gland

Thyroid gland

Soft palate

Cutaneous malignancy

T category

T0

$\mathrm{T} 1$

$\mathrm{T} 1$

T3

$\mathrm{T} 4 \mathrm{a}$

$\mathrm{T} 4 \mathrm{~b}$

$\mathrm{N}$ category

N0

N1

N2a

$\mathrm{N} 2 \mathrm{~b}$

$\mathrm{N} 2 \mathrm{c}$

N3a

$\mathrm{N} 3 \mathrm{~b}$

ECOG performance status

0

11-point modified frailty index

0

1

Procedures performed

$1 \mathrm{Hemi} /$ partial glossectomy with nd

2 Near total glossectomy with mm with nd

3 Superficial parotidectomy

4 Extended radical parotidectomy

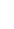

(1)

(1)

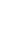


Table 1 (continued)

5 Maxillectomy 3

6 Buccal mucosa composite resection with $\mathrm{mm} / \mathrm{sm}$ and UA $\quad 14$

7 Orbital exenteration 1

8 Total laryngectomy 3

9 Secondary suturing and tongue flap 1

10 Secondary suturing and stsg 1

11 Salvage neck dissection 1

12 Total thyroidectomy

13 Tracheostomy 1

Reconstruction

1 Infrahyoid flap $\quad 2$

2 Supraclavicular flap $\quad 2$

3 Tongue flap 1

4 PMMF 14

5 Bipaddle PMMF 1

6 Masseter rotation 1

7 Mustarde flap 1

8 Nasolabial flap 1

Airway management

Preoperative

Tracheostomy

Postoperative

Tracheostomy 30

Postoperative outcome

30-day mortality

Clavien-Dindo score

$3 \mathrm{a}-2$

$3 \mathrm{~b}-1$

Complications

Aspiration pneumonia 4

Chyle leak 2

Hyponatremia $\quad 2$

Flap loss

Secondary haemorrhage $\quad 2$

Seroma

Wound dehiscence

Time to initiation of oral feeds(days) $\quad 7.35(2-30)$

Time to decannulation (days)

Positive COVID-19 status at surgery $(n=8)$

Indications

COVID-19 ARDS tracheostomy

Infrastructural maxillectomy (invasive sino-orbital

mucormycosis)

1

Incision and drainage parotid abscess

Comorbidities

Diabetes

Tuberculosis

Hypertension 
Table 1 (continued)

\begin{tabular}{ll}
\hline Coronary artery disease & 1 \\
Fungal pneumonia & 2 \\
Duration of intubation prior to tracheostomy & 5.75 days \\
& (range \\
& $3-8$ days) \\
\hline
\end{tabular}

Abbreviations: $G A$ general anaesthesia, $L A$ local anaesthesia, $n d$ neck dissection, $m m$ marginal mandibulectomy, sm segmental mandibulectomy, ua upper alveolectomy, stsg split-thickness skin graft, $P M M F$ pectoralis major myocutaneous flap, $A R D S$ acute respiratory distress syndrome

(asymptomatic COVID-19). Of these, one patient with carcinoma buccal mucosa T4bN2aM0 developed progressive disease during the period of isolation and was deemed inoperable at re-assessment. The second patient with carcinoma tongue T3NOM0 was advised home isolation and was lost to follow-up. None of the patients with unknown COVID19 status posted for emergency procedures were found to be positive on the preoperative RT-PCR. During the entire duration of hospital stay, all the patients who had undergone elective or emergency procedures with baseline negative RT-PCR remained COVID-19-negative. Similarly, no case of COVID-19 was reported among the health-care workers involved in the team managing these patients.

\section{Elective Procedures with Baseline Negative RT-PCR}

This cohort included 40 diagnostic and 41 ablative and/ or reconstructive head and neck procedures. Biopsy under general anaesthesia was preferred for the larynx and the hypopharynx $(n=9)$. Biopsy for tumours involving the oral cavity and oropharynx were performed under local anaesthesia and sedation. All diagnostic procedures were completed in a day-care set-up.

Forty-one definitive head and neck surgical procedures were undertaken on 39 patients. Two patients required return to the operating room for wound dehiscence (repair of the floor of mouth defect with tongue flap and external skin defect by cervical rotation, 1; repair of posterior auricular skin defect with split-thickness skin graft, 1). Baseline characteristics have been summarised in Table 1. To reduce operative time and the total number of personnel involved as well as given the resource-constrained situation, pectoralis major myocutaneous flap and other regional flaps were given preference over microvascular reconstruction. Preference was given to locally advanced tumours that were likely to become unresectable with a waiting period of 3 weeks. Due to constraints imposed by the shunting of intensive care units towards care of critically COVID-19 patients, postoperative airway management following composite head and neck cancer resection and reconstruction had to be shifted from overnight ICU stay in an intubated state towards elective tracheostomy. Elective tracheostomy minimised the need of postoperative sedation and mandatory ventilatory support, and we could encourage early ambulation by shifting the patient to T-piece or room air on the morning of the first postoperative day itself. The theoretical risk of increased COVID-19 transmission in tracheostomised patients was kept in check by the use of heat-moisture exchange device, subglottic suction device and use of second-level PPE by all health-care workers involved in patient care [8].

\section{Perioperative Outcome}

There were no reports of 30-day mortality. Two patients required 30-day readmission. One was a case of carcinoma buccal mucosa $\mathrm{T} 4 \mathrm{aN} 2 \mathrm{aM} 0$ post segmental mandibulectomy, upper alveolectomy, modified radical neck dissection type II and reconstruction with pectoralis major myocutaneous flap with delayed partial flap loss and floor of mouth defect. The second readmission was in a patient who presented with minor peristomal bleed following total laryngectomy which was managed conservatively. Complications encountered and Clavien-Dindo grading has been presented in Table 1.

\section{Unknown COVID-19 Status at Surgery}

This cohort included patients with life-threatening head and neck emergency. All 11 procedures performed were tracheostomies under local anaesthesia for upper airway obstruction secondary to head and neck malignancy.

\section{Positive COVID-19 Status at Surgery}

Tracheostomy under general anaesthesia for anticipated prolonged intubation in patients with COVID-19 ARDS was the most common indication $(n=6)$ (Table 1$)$. Infrastructural maxillectomy was performed in a patient with acute invasive sino-orbital mucormycosis. The patient was initially given a therapeutic trial of liposomal amphotericin B and Posaconazole. Following stabilisation of his COVID-19 pneumonia and regression of disease from the orbit, limited debridement in the form of infrastructural maxillectomy was performed under general anaesthesia. The patient was orally intubated before surgery and was electively tracheostomised at the end of the procedure. The second procedure 
was incision and drainage for parotid abscess performed under local anaesthesia.

\section{Matched Pair Analysis with Retrospective Cohort Operated before COVID-19 Pandemic}

Post-hoc analysis was performed to compare perioperative complications encountered during the pandemic with those experienced before COVID-19. For this analysis, patients from the study cohort who had undergone major head and neck surgery (RT-PCR negative) for head and neck cancer ( $n=38,1$ patient of pleomorphic adenoma excluded) were included. This group was compared with a historical cohort of similar patients who had undergone surgery from January to December $2016(n=111)$. Table 2 provides baseline differences between the two cohorts. Significant differences

Table 2 Baseline characteristics and differences in complication (Clavien-Dindo Score) of the RT-PCR negative major head and surgery arm with pre-pandemic cohort

\begin{tabular}{|c|c|c|c|}
\hline Variable & $\begin{array}{l}\text { RT-PCR negative major head and } \\
\text { neck surgery arm }(n=38)\end{array}$ & Pre-pandemic cohort $(n=111)$ & $P(95 \%$ Confidence Interval) \\
\hline Mean Age (+/-SD) (Years) & $48.0(11.2)$ & $48.1(13.5)$ & $0.48(-4.7$ to 4.9$)$ \\
\hline Sex & & & 0.76 \\
\hline Male & 30 & 85 & \\
\hline Female & 8 & 26 & \\
\hline Subsite & & & 0.78 \\
\hline $\begin{array}{l}\text { Upper aerodigestive tract (oral cav- } \\
\text { ity, oropharynx, larynx, paranasal } \\
\text { sinus) }\end{array}$ & 34 & 96 & \\
\hline Non-mucosal (Parotid, thyroid, skin) & 4 & 15 & \\
\hline Stage & & & 0.01 \\
\hline Early (Stage I\&II) & 8 & 8 & \\
\hline Advanced (Stage III \&IV) & 30 & 103 & \\
\hline Surgery & & & 0.11 \\
\hline $\begin{array}{l}\text { Transoral resection with no recon- } \\
\text { struction }\end{array}$ & 11 & 40 & \\
\hline $\begin{array}{l}\text { Composite resection with } \\
\text { reconstruction(includes maxillec- } \\
\text { tomy and laryngectomy) }\end{array}$ & 24 & 50 & \\
\hline $\begin{array}{l}\text { Extramucosal surgery (thyroid/ } \\
\text { parotid/skin) }\end{array}$ & 3 & 21 & \\
\hline Modified Frailty Index Score & & & $<0.001$ \\
\hline 0 & 19 & 92 & \\
\hline 1 & 14 & 16 & \\
\hline 2 & 5 & 3 & \\
\hline \multicolumn{4}{|l|}{ Reconstruction } \\
\hline None & 15 & 76 & 0.002 \\
\hline Pedicled & 23 & 25 & $<0.001$ \\
\hline Free-Flap & 0 & 10 & \\
\hline Clavien-Dindo Score & 2031221 & 43320396 & 0.130 .170 .080 .0020 .49 \\
\hline 0 & 20 & 43 & 0.13 \\
\hline 1 & 3 & 3 & 0.17 \\
\hline 2 & 12 & 20 & 0.08 \\
\hline 3 & 2 & 39 & 0.002 \\
\hline 4 & 1 & 6 & 0.49 \\
\hline Detail of Complications & $\begin{array}{l}\text { Aspiration -4pneumoniaChyle } \\
\text { leak-2Hyponatremia-2Flap loss- } \\
\text { 5Secondary hemorrhage2Seroma- } \\
\text { 1Wound dehiscence-1 }\end{array}$ & $\begin{array}{l}\text { AspirationPneumonia:6Flap Loss } \\
\text { and salivary fistula-13Hematoma- } \\
\text { 4Wound Gape-13Hypocalcemia- } \\
\text { 10Cord palsy-4Others -10 }\end{array}$ & \\
\hline $\begin{array}{l}\text { Complications requiring surgical } \\
\text { intervention }\end{array}$ & 2 & 10 & \\
\hline
\end{tabular}


were noted between the two groups in terms of distribution of stage of the tumour and modified frailty index. Another aspect of difference noted was the reconstructive surgery performed. Though number of pedicled flaps performed during the pandemic outnumbered those performed during the pre-pandemic time-period, no microvascular reconstruction was performed in the former. The unmatched analysis for Clavien-Dindo score did not reveal increase rates of complications in the pandemic cohort.

A further matched-pair analysis was planned (Table 3) using propensity score matching. 1:1 matching was performed using the logit regression model. This gave rise to fully balanced 32 pairs in each group (total $n=64$ ). The variables which were considered for matching have been highlighted in Table 3. The pandemic cohort demonstrated increased number of patients with lower Clavien-Dindo score. Whereas the pre-pandemic cohort demonstrated greater number of patients with higher Clavien-Dindo scores. This difference was found to be statistically significant. Therefore, with similarly staged tumours and with similar degree of coexisting comorbid illness, no unusual increase in perioperative complications were noted in those patients operated during the COVID-19 pandemic provided pre-operative RT-PCR was negative.

\section{Discussion}

This study was conducted at a dedicated oncology referral centre in India, which has been transformed into a COVIDdesignated treatment facility. Since the emergence of the pandemic, 4662 COVID-19 cases have been treated until the end of the study duration. Following the 21-day stateimposed lockdown announced on 24th March, 2020, oncology services at our Institute had to be suspended. Subsequently, oncology facilities were reinstated in a scaled-down fashion to strike a balance between the steeply rising curves of COVID-19 cases as well as to deliver cancer care without compromising survival and transmission of COVID-19 among cancer patients and health-care workers. We had limited infrastructure at our disposal — a four bedded isolation cubicle and 20 bedded emergency wards which were shared between 6 clinical branches working for cancer patient care. In this study, we have highlighted our Institutional practice
Table 3 Matched-Pair analysis between COVID-19 and PreCOVID-19 subset with ClavienDindo score as the outcome

\begin{tabular}{|c|c|c|c|}
\hline Variable & $\begin{array}{l}\text { RT-PCR negative major head } \\
\text { and neck surgery arm }(\mathrm{n}=32)\end{array}$ & $\begin{array}{l}\text { Pre-Pandemic } \\
\text { Cohort }(\mathrm{n}=32)\end{array}$ & $\mathrm{p}$ \\
\hline Mean Age (+/-SD) (Years) & $48.5(11.5)$ & $46.18(12.1)$ & 0.78 \\
\hline Sex & & & 0.75 \\
\hline Male & 25 & 27 & \\
\hline Female & 7 & 5 & \\
\hline Subsite & & & 0.39 \\
\hline $\begin{array}{l}\text { Upper aerodigestive tract (oral cavity, oro- } \\
\text { pharynx, larynx, paranasal sinus) }\end{array}$ & 28 & 30 & \\
\hline Non-mucosal (Parotid, thyroid, skin) & 4 & 2 & \\
\hline Stage & & & 0.71 \\
\hline Early (Stage I\&II) & 5 & 4 & \\
\hline Advanced (Stage III \&IV) & 27 & 28 & \\
\hline Surgery & & & 0.44 \\
\hline Transoral resection with no reconstruction & 9 & 12 & \\
\hline $\begin{array}{l}\text { Composite resection with } \\
\text { reconstruction(includes maxillectomy and } \\
\text { laryngectomy) }\end{array}$ & 20 & 15 & \\
\hline Extramucosal surgery (thyroid/parotid/skin) & 3 & 5 & \\
\hline Modified Frailty Score & & & 0.88 \\
\hline 0 & 19 & 19 & \\
\hline 1 & 11 & 10 & \\
\hline 2 & 2 & 3 & \\
\hline \multicolumn{4}{|l|}{ Clavien-Dindo Score } \\
\hline 0 & 17 & 18 & \\
\hline 1 & 2 & 0 & \\
\hline 2 & 10 & 2 & \\
\hline 3 & 2 & 9 & 0.007 \\
\hline 4 & 1 & 3 & \\
\hline
\end{tabular}


of COVID-19 screening and testing strategy and presented our experience on 100 head and neck procedures performed over 21 weeks. Of the 92 procedures performed on COVID19-negative patients [COVID-19 status at surgery: known, 81 (elective); unknown, 11 (emergent)] in accordance with our institutional management guidelines, none of the patients or health-care workers developed symptoms consistent with COVID-19 during the duration of the hospital stay. 30-day mortality, 30-day readmission and complication rates were $0 \%, 2.17 \%$ and $18.4 \%$, respectively. This series also included 6 tracheostomies, maxillectomy for invasive mucormycosis $(n=1)$ and incision and drainage of parotid abscess $(n=1)$ in known COVID-19-positive patients. For COVID-19 ARDS tracheostomies, the decision for tracheostomy was taken by the ICU team based on the ventilatory requirements and patients response to therapy. Similarly, for any emergency procedures on COVID-19-positive patients, maximum therapeutic trial with available non-surgical modalities was offered (dual-antifungal therapy for invasive mucormycosis and culture-directed antibiotics and bed-side aspiration for abscess) to minimise viral load and thus the risk of infection. The decision towards early surgical intervention should be taken in patients who develop progressive symptoms refractory to medical management. Additionally, a matched pair analysis with a historical pre-pandemic cohort did not reveal increased rates of complications in those patients operated during the pandemic.

A matched-pair analysis comparing postoperative outcomes in patients with the perioperative diagnosis of COVID-19 $(n=41)$ with those with COVID-19-negative status $(n=123)$ revealed higher 30 -day mortality $(O R, 9.5)$, greater incidence of postoperative complications, especially pulmonary complications $(O R, 35.6)$ and thrombotic complications $(O R, 13.2)$ in COVID-19-positive cohort [9]. A recently concluded systematic review collating the results across 4 studies reporting on the postoperative outcomes of asymptomatic COVID-19-positive patients, revealed a pooled postoperative severe complication rate of $27.5 \%$, predominantly pulmonary complications and universal transmission of COVID-19 among health-care workers [10]. Patients with HNC are considered to be highly vulnerable to the adverse effects of COVID-19 infection in the perioperative period given the advanced age of presentation, association with smoking, presence of multiple comorbidities and immunosuppression secondary to radiation or chemoradiation [11]. This provides robust evidence in favour of preoperative screening and screening before aerosol generating procedures. Such a directive was unavailable in the initial part of the pandemic [12]. The Indian Council of Medical Research (ICMR) expanded the existing guidelines for RTPCR to include asymptomatic individuals planned for elective surgeries and aerosol-generating procedures on $23 \mathrm{rd}$ June 2020 [13].
Results of a survey conducted in India on the impact of COVID-19 pandemic on HNC care were published in April 2020 and revealed that $69 \%$ of the dedicated cancer centres across India had halted elective procedures and restricted themselves to emergency procedures. For HNC, treatment should ideally begin within 30 days of diagnosis and no later than 60 days, to avoid compromising survival outcomes [14-16]. A 2-4-week delay results in a $68 \%$ increase in the tumour volume and a 4-week delay results in a $70 \%$ increase, with the potential risk of being rendered unresectable [17]. Though the aftermath of the pandemic is largely unknown, the COVIDSurg Collaborative had predicted that 28404603 surgeries (37.7\% cancer surgeries) would be cancelled in the first 12 weeks of the pandemic [18]. Even after ramping up of oncology facilities by $20 \%$, it would take 45 weeks to clear the backlog [18]. Therefore, the continuation of oncology services would be the most pragmatic approach.

Numerous scientific bodies across the world have put forth guidelines and directives for practice modifications for the safe continuation of HNC care [5]. Though there is contention surrounding the duration of deferral of early HNC and use of neoadjuvant chemotherapy or metronomic chemotherapy to tide over the waiting period, the overall theme continues to be the continuation of oncology services with preoperative COVID-19 testing and use of appropriate PPE $[5,19]$. For judicious use of resources, a well-validated triage system is the need of the hour. The group from the USA headed by Morrison et al. classified advanced oral cavity, oropharyngeal, laryngeal malignancies and tumours including aggressive thyroid malignancies causing airway narrowing as urgent (waiting time $<2$ weeks), semi-urgent included moderately advanced oral cavity, oropharyngeal and laryngeal malignancies, skull base malignancies, aggressive thyroid tumours and salivary gland tumours with a recommended waiting period of 2-4 weeks. Early upperaerodigestive tract tumours, well-differentiated thyroid cancers and indolent salivary gland cancers were categorized as less urgent and can be operated upon after a waiting period of 4 weeks [20]. de-Almeida et al. have designed SPARTAN-HN (Surgical Prioritization and Ranking Tool and Navigation Aid for Head and Neck Cancer) which is a scoring tool that takes into account the following parameters: performance status, comorbidities, stage, histology, prior radiotherapy, alternate treatment availability, the potential for tumour progression, extent and expected length of surgery, expected hospital duration, need for intensive care support and waiting-time target [21].

The results that have been presented this study suggest deviation from the existing standard of care. Firstly, for patients undergoing composite resection involving mandible, upper alveolus with concomitant neck dissection and reconstruction, tracheostomy has been performed instead of overnight intubation in an intensive care unit to avoid overwhelming the critical care 
Table 4 Review of literature on the outcomes following head and neck surgery during COVID-19 pandemic

\begin{tabular}{|c|c|c|c|}
\hline Author & $n$ & Screening strategy & Outcome \\
\hline Brar S [24] & 47 & $\begin{array}{l}47 \% \text {, negative swab and CXR } \\
81 \% \text {, negative swab or CXR } \\
19 \% \text {, no swab or CXR }\end{array}$ & $\begin{array}{l}\text { - No 30-day mortality } \\
\text { - Clavien-Dindo: } \\
1-2 \\
2-4 \\
3-1 \\
\text { - No conversion to COVID-19-positive status during hospital stay }\end{array}$ \\
\hline Mueller SK [25] & $\begin{array}{l}\text { Panendos- } \\
\text { copy, } 170 \\
\text { Ablative, } 189\end{array}$ & RT-PCR & $\begin{array}{l}\text { - No conversion to COVID-19-positive status during hospital stay } \\
\text { - Perioperative outcomes: N/A }\end{array}$ \\
\hline Valentini V [26] & 56 & RT-PCR, telephonic triage & N/A \\
\hline Riva G [27] & 19 & RT-PCR,CXR, telephonic triage & $\begin{array}{l}\text { - No conversion to COVID-19-positive status during hospital stay } \\
\text { - Perioperative outcomes: N/A }\end{array}$ \\
\hline Galli J [28] & 27 & RT-PCR, CXR or CT chest & $\begin{array}{l}\text { - No conversion to COVID-19-positive status during hospital stay } \\
\text { - Perioperative outcomes: N/A }\end{array}$ \\
\hline
\end{tabular}

Abbreviation: $C X R$ chest X-ray

facilities and manpower. Secondly, we have preferred the use of regional and loco-regional flaps instead of microvascular reconstruction. The latter requires close monitoring in ICU set up, prolonged operative and need for two team approaches which translates into overcrowding of the operating room. Microvascular reconstruction continues to be the standard of care at a few centres with adequate resources. However, pedicled flaps being the work-horse can be considered in present circumstances where patients may be managed outside an intensive or high dependency unit with rapid turnover [22].

Our study shows that the " $4 \mathrm{R}$ " principle in the management of head and neck cancer can be easily followed even during this pandemic. Resection with adequate margins with complete oncological clearance is always possible. Tailoring reconstruction according to the needs. Rehabilitate and look for future recurrences through the help of telemedicine follow-up services [23]. This study, however, does not provide data related to stage migration in HNC patients awaiting surgery during the period when elective surgeries were deferred.

We have compared the results of our study with other publications reporting on the surgical outcomes in HNC operated during the period of the pandemic in Table 4 [24-28]. Currently, we only have single institution experience reporting on retrospective data. However, the data available does instil confidence in continuing HNC care in the midst of a pandemic on a cohort of patients considered to be the most vulnerable and high-risk for disease transmission even among health-care workers.

\section{Conclusion}

We have elaborated on our retrospective data on head and neck procedures performed in an oncology centre which also serves as a COVID-designated treatment facility. Adhering to the screening and patient workflow guidelines in place and adequate safety measures for health-care workers, it is feasible and safe to continue HNC care.

Acknowledgements We would like to thank Dr Vishwajeet Singh (Biostatistics) from the Department of Geriatric Medicine, A.I.I.M.S, New Delhi, for his contribution to the matched pair analysis presented in this study.

Author Contribution Conceptualization: SP, SV, CAS. Methodology: SP, SV, CAS, PS

Formal analysis and investigation: SP, AB, AT, PS

Writing (original draft preparation): SP, AB, KS, RK

Writing (review and editing): PT, VPM, RG, SB, KT

Supervision: AT, AM, SB

Data Availability Datasheet pertaining to this study can be made available upon communicating with the corresponding author.

Code Availability Not applicable

\section{Declarations}

Ethics Approval This retrospective review was exempted from Ethical Clearance by the Institutional Ethical Committee. The study was performed in accordance with the ethical standards as laid down in the 1964 Declaration of Helsinki. Informed written consent was obtained from all the participants.

Consent to Participate and Consent for Publication Consent was obtained from all the participants.

Conflict of Interest The authors declare no competing interests.

\section{References}

1. Timeline: WHO's COVID-19 response [Internet]. [cited 2020 Sep 30]. Available from: https://www.who.int/emergencies/diseases/ novel-coronavirus-2019/interactive-timeline. Accessed 30 Sept 2020 
2. Oncology TL (2020) Safeguarding cancer care in a postCOVID-19 world. Lancet Oncol 21:603

3. Bhattacharjee A, Chakraborty A, Purkaystha P (2006) Prevalence of head and neck cancers in the north east - an institutional study. Indian J Otolaryngol Head Neck Surg 8:15-19

4. Day AT, Sher DJ, Lee RC, Truelson JM, Myers LL, Sumer BD, Stankova L, Tillman BN, Hughes RS, Khan SA, Gordin EA (2020) Head and neck oncology during the COVID-19 pandemic: Reconsidering traditional treatment paradigms in light of new surgical and other multilevel risks. Oral Oncol 105:104684. https:// doi.org/10.1016/j.oraloncology.2020.104684

5. Garg PK, Kaul P, Choudhary D et al (2020) Discordance of COVID-19 guidelines for patients with cancer: a systematic review. J Surg Oncol. https://doi.org/10.1002/jso.26110

6. Adams P, Ghanem T, Stachler R, Hall F, Velanovich V, Rubinfeld I (2013) Frailty as a predictor of morbidity and mortality in inpatient head and neck surgery. JAMA Otolaryngol 139:783-789

7. Monteiro E, Sklar MC, Eskander A, de Almeida JR, Shrime M, Gullane P et al (2014) Assessment of the Clavien-Dindo classification system for complications in head and neck surgery. Laryngoscope 124:2726-2731

8. Bindra A, Kajal S, Sikka K (2020) Tracheostomy during COVID19 pandemic: Viewpoint. J Neuroanaesth Crit Care Sep. https:// doi.org/10.1055/s-0040-1716428

9. Doglietto F, Vezzoli M, Gheza F, Lussardi GL, Domenicucci M, Vecchiarelli L et al (2020) Factors associated with surgical mortality and complications among patients with and without Coronavirus Disease 2019 (COVID-19) in Italy. JAMA Surg 155(8):691-702. https://doi.org/10.1001/jamasurg.2020.2713

10. Nahshon C, Bitterman A, Haddad R, Hazzan D, Lavie O (2020) Hazardous postoperative outcomes of unexpected covid-19 infected patients: a call for global consideration of sampling all asymptomatic patients before surgical treatment. World J Surg 44:2477-2481

11. Werner MT, Carey RM, Albergotti WG, Lukens JN, Brody RM (2020) Impact of the COVID-19 pandemic on the management of head and neck malignancies. Otolaryngol-Head Neck Surg Off J Am Acad Otolaryngol-Head Neck Surg 162:816-817

12. Gupta A, Arora V, Nair D, Agrawal N, Su YX, Holsinger FC, Chan JYK (2020) Status and strategies for the management of head and neck cancer during COVID-19 pandemic: Indian scenario. Head Neck 42(7):1460-1465. https://doi.org/10.1002/hed. 26227

13. Testing Strategy [Internet]. [cited 2020 Oct 4]. Available from: https://www.icmr.gov.in/cteststrat.html. Accessed 4 Oct 2020

14. Murphy CT, Galloway TJ, Handorf EA, Egleston BL, Wang LS, Mehra R et al (2016) Survival impact of increasing time to treatment initiation for patients with head and neck cancer in the United States. J Clin Oncol 34:169-178

15. Liao C-T, Chen H-N, Wen Y-W, Lee SR, Ng S-H, Liu T-W et al (2017) Association between the diagnosis-to-treatment interval and overall survival in Taiwanese patients with oral cavity squamous cell carcinoma. Eur J Cancer 72:226-234
16. Schutte HW, Heutink F, Wellenstein DJ, van den Broek GB, van den Hoogen FJA, Marres HAM et al (2020) Impact of time to diagnosis and treatment in head and neck cancer: a systematic review. Otolaryngol-Head Neck Surg 162:446-457

17. Wildt J, Bundgaard T, Bentzen SM (1995) Delay in the diagnosis of oral squamous cell carcinoma. Clin Otolaryngol 20:21-25

18. COVIDSurg Collaborative (2020) Elective surgery cancellations due to the COVID-19 pandemic: global predictive modelling to inform surgical recovery plans. Br J Surg 107(11):1440-1449. https://doi.org/10.1002/bjs.11746

19. Sultania M, Muduly D, Imaduddin M, Kar M (2020) Oral cancer surgery and covid pandemic - metronomic therapy shows a promising role while awaiting surgery. Oral Oncol 106:104814

20. Morrison DR, Gentile C, McCammon S, Buczek E (2020) Head and neck oncologic surgery in the covid-19 pandemic: our experience in a deep south tertiary care center. Head Neck 42:1471-1476

21. de Almeida JR, Noel CW, Forner D, Zhang H, Nichols AC, Cohen MA et al (2020) Development and validation of a surgical prioritization and ranking tool and navigation aid for head and neck cancer (SPARTAN-HN) in a scarce resource setting: response to the COVID-19 pandemic. Cancer 126(22):4895-4904. https://doi. org/10.1002/cncr.33114

22. Zaid W, Schlieve T (2020) The early effects of coronavirus disease-2019 on head and neck oncology and microvascular reconstruction practice: a national survey of oral and maxillofacial surgeons enrolled in the head and neck special interest group. J Oral Maxillofac Surg 78:1859-1868

23. Ohlstein JF, Garner J, Takashima M (2020) Telemedicine in otolaryngology in the COVID-19 era: initial lessons learned. Laryngoscope. 130(11):2568-2573. https://doi.org/10.1002/lary.29030

24. Brar S, Ofo E, Hyde N, Kim D, Odutoye T, Allin D et al (2020) Outcomes of elective head and neck confirmed or suspected cancer surgery during the COVID-19 pandemic. Eur Arch Otorhinolaryngol 15:1-6

25. Mueller SK, Traxdorf M, Mantsopoulos K et al (2021) Considerations for continuing semielective and emergency otolaryngological procedures during the COVID-19 pandemic. Ear, Nose \& Throat Journal 100(1):19-25. https://doi.org/10.1177/0145561320 952506

26. Valentini V, Pucci R, Battisti A, Cassoni A (2020) Head and neck cancer cannot wait for this pandemic to end: risks, challenges and perspectives of oral-maxillofacial surgeon during COVID-19. Oral Oncol 106:104758

27. Riva G, Pizzo C, Fassone E, Pecorari G (2020) Head and neck cancer surgery in covid-19 pandemic in Northern Italy. Oral Oncol 107:104835

28. Galli J, Settimi S, Tricarico L, Almadori G, Paludetti G (2020) Clinical and surgical management of patients with head and neck cancer in a covid-19 dedicated center in Italy. Head Neck 42:1466-1470

Publisher's Note Springer Nature remains neutral with regard to jurisdictional claims in published maps and institutional affiliations. 